\title{
ADIC-COMPLETION AND SOME DUAL HOMOLOGICAL RESULTS
}

\author{
ANNE-MARIE SIMON
}

To the memory of Pere Menal

\begin{abstract}
Let $a$ be an ideal of a commutative ring $A$. There is a kind of duality betweer the left derived functors $U_{i}^{a}$ of the a-adic completion functor, called local homology functors, and the local cohomology functors $H_{\mathbf{a}}^{i}$.

Some dual results are obtained for these $U_{i}^{a}$, and also inequalities involving both loced homology and local cohomology when the ring $A$ is noetherian or more generally when the $U^{2}$ and $H_{a}$-global dimensious of $A$ are finite.
\end{abstract}

In this paper $A$ is a commutative ring, $a$ an ideal of $A$ and the $A$ modules are given the a-adic topology.

There is a certain duality between the left derived functors $U_{i}^{a}$ of the a-adic completion functor and the local cohomology functors $H_{t+1}^{i}$ first observed by Matlis when the ideal $a$ is generated by a (finite) regular sequence, true also for any noetherian ring. More recently, that duality has also been observed by Greenlecs and May in a more general context.

The purpose of this note is to pursue the analogy between the local cohomology functors and these functors $U_{i}^{a}$, called local homology functors by Greenlees and May.

First we have dual results about codepth, a notion dual to the notion of homological depth or grade.

To go further, we need some noetherian hypothesis in order to have a change of rirys theorem for the $U_{i}^{o}$, analogous to the corresponding one in local cohomology. This brings us back to the first case studied by Matlis, namely the case of an ideal generated by a regular sequence, and allows generalizations of some Matlis results. As a consequence, we obtain varishing results for the $U_{i}^{a}$, and also inequalities involving 
both local cohomology and local homology. So local cohomology and local homology are not only duals of each other, but also intimately connected.

As general references for commutative algebra and homological questions, we quote $[\mathbf{1}],[\mathbf{1 8}]$.

The work below has been communicated at the International workshop on local cohomology, geometric applications and related topics. We take this opportunity to thank the organizers and the participants for useful discussions.

\section{Preliminaries and notations}

In this first section we fix notations and collect the material we need. Though part of it appeared in different places, we think it is more convenient to have it at hand.

\subsection{Completion.}

Let $a$ be an ideal of the commutative ring $A$. The $A$-module are given the $a$-adic topology. The completion of an $A$-module $M$ is denoted by $\hat{M}$ : thus $\hat{M}=\underset{\leftarrow}{\lim M / a^{n} M}$. Let $\tau_{M}: M \rightarrow \hat{M}$ be the natural morphism.

Here and in the next section, the ideal $a$ is not necessarily finitely generated, and it might happen that the $A$-module $\hat{M}$, complete in its natural topology, is not complete in its a-adic topology. An example of this can be found in ([5, III, Section 2, exercise 12]) or in ([3, I, Section 3)).

Recall however the following result ([3, theorem 1.3.1], or [13, theoren $15]$, or $[\mathbf{1 8}, 2.2 .5])$.

Theorem. Suppose the ideal a finitely generated. Let $M$ be an $A$ module and $b$ an open ideal in the a-adic topology of $A$. Then the morphism $\tau_{M} \otimes A / b: M / b M \rightarrow \hat{M} / b \hat{M}$ is an isomorphism. So $\hat{M}$ is complete in its a-adic topology.

\subsection{When $\hat{f}$ is onto.}

The $a$-adic completion functor, though not right exact, preserves surjection. However, we want to know precisely when the completion of a morphism is onto. The following lemma was proved in $([\mathbf{1 6}, 1.2])$ for a noetherian ring $A$, using 1.1. It is true in general.

Lemma. Let $f: M \rightarrow N$ be a morphism of $A$-modules. Then $\hat{f}$ is surjective if and only if $N=f M+a N$. 
Proof: If $N=f M+a N$, then $N=f M+a^{n} N$ for all $n \geq 1$, and the projective system of sequences

$$
0 \longrightarrow f^{-1}\left(a^{n} N\right) / a^{n} M \longrightarrow M / a^{n} M \longrightarrow N / a^{n} N \longrightarrow 0
$$

is exact. It is easily seen to be surjective (see $[16,1.2]$ ). So, after taking limits, we get an exact sequence and $\hat{f}$ is surjective.

Conversely, if $\hat{f}$ is surjective, we tensor the commutative natural diagram

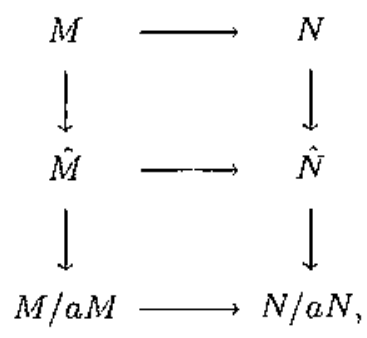

where the vertical composite maps are the natural projections, with $A / a$. The composite vertical maps become the identity, so $M / a M \rightarrow N / a N$ is surjective and $N=\int M+a N$.

\subsection{The left derived functors of the completion functor.}

The left derived functors of the a-adic completion functor are denoted by $U_{i}^{2}$. These were first studied by Matlis when the ideal is generated by a finite regular sequence [12], [13]. We used them in [16], where the ring is noetherian. More recently, they have been cornputed by Greenlees and May in a more general situation [7].

Let $L_{1} \stackrel{f}{\rightarrow} L_{0} \rightarrow M \rightarrow 0$ be an exact sequence, with $L_{0}, L_{1}$ free. By definition $U_{0}^{a}(M)=\operatorname{coker} \hat{f}$, so we have natural morphisms $M \rightarrow$ $U_{0}^{a}(M) \rightarrow \hat{M}$ whose composite is $\tau_{M}$. The following lemma $([16,5.1])$, consequence of 1.2 , is still available.

\section{Lemma.}

(i) The natural morphism $U_{0}^{\alpha}(M) \rightarrow \hat{M}$ is onto.

(ii) $\hat{M}=0$ if and only if $M=a M$ if and only if $U_{0}^{a}(M)=0$.

(iii) If the ideal a is finitely generated, then $\left(U_{0}^{\prime \prime}(M)\right)^{\wedge}=\hat{M}$.

\subsection{The class $\mathcal{C}_{a}$.}

Let $C_{a}$ be the class of modules $M$ such that $U_{0}^{a}(M)=\bar{M}$ and $U_{i}^{a}(M)=$ 0 for $i>0$. A standard homological argument shows that $U_{i}^{a}(M)$ can be computed using a left resolution of $M$ with modules in $C_{a}$, and it is worthwile to note that, flat modules belong to $C_{a 2}$. 
More generally, let $a_{i_{2}}, n>0$, be a decreasing sequence of ideals which form a basis of the a-adic topology. A module $M$ such that $\operatorname{Tor}_{i}^{A}\left(A / a_{n}, M\right)=0$ for all $i>0$ and all $n>0$ belongs to $\mathcal{C}_{a}$ ([12, corollary 4.5]).

When the ring is noctherian, the completion of a free module is flat ([14, p. 77], or $[3,4,7]$, or $[18,2.2 .4])$. This can be used to show that complete modules belong to $C_{a}$ when $A$ is noetherian ([16,5.2j).

\subsection{Local cohomology and Matlis duality.}

Recall the functor $H_{a}^{0}: H_{a}^{0}(M)=\left\{x \in M \mid a^{n} x=0\right.$ for sorne natural number $n\}$, whose right derived functors $H_{a}^{i}$ are the local cohomology functors.

Recall also the Matlis duality. Let $E$ be the injective hull of the direct sum of all the $A / m$ with $m$ a maximal ideal of $A$. The Matlis duality functor, defined by $M^{\vee}=\operatorname{Hom}_{A}(M, E)$, is faithfully exact [12], [13], and we have the Ext-Tor duality:

$$
\operatorname{Tor}_{i}^{A}(N, M)^{\vee} \simeq \operatorname{Ext}_{A}^{i}\left(N, M^{\vee}\right)
$$

when $N$ has a projective resclution composed of finitely gencrated modules $([6, V I, 5.1,5.3])$ :

$$
\operatorname{Tor}_{i}^{A}\left(N, M^{\vee}\right) \simeq \operatorname{Ext}_{A}^{i}(N, M)^{\vee} .
$$

When the ring is noetherian, $H_{a}^{0}(M)^{\vee} \simeq\left(M^{\vee}\right)^{\wedge}$ and $H_{a}^{i}(M)^{\vee} \simeq$ $U_{i}^{a}\left(M^{\vee}\right)$ for all $i([16,4.2,5.6])$. This is based on the fact that, over a noetherian ring, fat modules and injective modules are interchanged by Matlis duality. This was first proved by Matlis when the ideal $a$ is generated by a regular sequence.

But modules are not necessarily duals, so informations about the local cohomology functors $H_{a}^{i}$ do not always provide informations about the local homology functors $U_{i}^{u}$.

\subsection{Formal depth, codepth and dimension.}

A sequence of covariant additive functors $G_{n}: \mathcal{A} \rightarrow \mathcal{B}, n \in Z$, between abelian categories is a descending connected exact sequence of functors if $G_{n}=0$ for $n<0$ and if each exact sequence $0 \rightarrow M^{\prime} \rightarrow M \rightarrow M^{\prime \prime} \rightarrow 0$ in $\mathcal{A}$ gives rise to a long exact sequence

$$
\cdots \longrightarrow G_{i+1}\left(M^{\prime \prime}\right) \longrightarrow G_{i}\left(M^{\prime}\right) \longrightarrow G_{n}(M) \longrightarrow G_{i}\left(M^{\prime \prime}\right) \longrightarrow \ldots
$$

in a ftuctorial way. 
When we have such a sequence, as in $([18,1.1]$ or $[17])$, we put $g-(M)=\inf \left\{i G_{i}(M) \neq o\right\}$ for each object $M$ in $\mathcal{A}$ (so that $o \leq$ $\left.g_{-}(M) \leq \infty\right)$.

Dually, we define $f^{-}(M)$ for an ascending connected exact sequence of functors $F^{n}$ in the same way.

'These numbers can be viewed as a kind of codepth or depth respectively.

When $a$ is an ideal of the ring $A$, we are merely concerned with the sequence $\operatorname{Ext}_{A}^{i}(A / a, \cdot), \operatorname{Tor}_{i}^{A}(A / a, \cdot), H_{c}^{i}(\cdot), U_{i}^{c}(\cdot)$ and with the corresponding numbers. Here are some first renarks about them, which will be completed later $(1.7,2.4)$.

Proposition. Let $a \subset b$ be ideals in the ring $A$, let $M$ an $A$-module

(i) $\operatorname{ext}_{A}^{-}(A / a, M)=h_{a}^{-}(M)$

(ii) $\operatorname{tor}_{-}^{A}(A / a, M)=\operatorname{ext}_{A}^{-}\left(A / a, M^{\vee}\right)$

(iii) $\operatorname{ext}_{A}^{-}(A / a, M) \leq \operatorname{ext}_{A}^{-}(A / b, M)$

(iv) $\operatorname{tor}_{-}^{A}(A / a, M) \leq \operatorname{tor}_{-}^{A}(A / b, M)$

(v) the numbers $\operatorname{ext}_{A}^{-}(A / a, M), \operatorname{tor}_{-}^{A}(A / a, M)$ only depend on the topology defined by the ideal a.

For (i) and (iii), see $([18,5.3 .15,5.3 .11])$; (ii) is a direct consequence of the Ext-Tor duality 1.5; (iv) follows from (ii) and (iii). Since local cohomology only depends on the topology defined by the ideal $a$, so does $\operatorname{ext}_{-}^{A}(A / a, \cdot)$ in view of (i), and so does also $\operatorname{tor}_{-}^{A}(A / a, \cdot)$ in view of (ii).

We also define $g_{+}(M)=\sup \left\{i \mid G_{i}(M) \neq 0\right\}$ (so that $g_{+}(M)=-\infty$ or $\left.0 \leq g_{+}(M) \leq \infty\right)$ and $f^{+}(M)$ in the same way. These last numbers are relative homological dimensions.

\subsection{The depth-codepth sensitivity of the Koszul complex.}

The depth sensitivity of the Koszul complex was proved by Barger and Hochster for a colnerent ring $[2]$, [8], by Kirby and Mehran for any commutative ring $[\mathbf{1 0}]$.

An approach involving both depth and codeptli can be found in ([18, 6.1] or [17]).

Let $x=x_{1}, \ldots, x_{n}$ be a sequence of clements of the ring $A$ generating an ideal $n$, and let $K .(x)$ be the associated Koszul complex. For an A-module $M$, we consider the desconding and ascending Koszal complexes $K .(x, M)=K .(x) \otimes_{A} M, K(x, M)=\operatorname{Hom}_{A}(K(x), M)$; we note their homologies by $H_{i}(x, M)$ and $H^{i}(x, M)$ respectively. These functors $H_{i}(x, \cdot)$ and $H^{i}(x, \cdot)$ are descending; ascending connected exact sequences of functors. In the notalions of 1.6 , we have the following result $([18,6.1 .6,6.1 .7])$. 
Theorem. Let $x=x_{1}, \ldots, x_{n}$ generate an ideal $a$ in the ring $A$ and let $M$ be an $A$-module. Then $h_{-}(x, M)=\operatorname{tor}_{-}^{A}(A / a, M), h^{-}(x, M)=$ $\operatorname{ext}_{A}^{-}(A / a, M)$.

Corollary. Let $a=\left(x_{1}, \ldots, x_{n}\right)$ be a finitely generated ideal of the ring $A$, and let $M$ be an $A$-module.

(i) $\operatorname{tor}_{-}^{A}\left(A / a, M^{\vee}\right)=\operatorname{ext}_{A}^{-}(A / a, M)$

(ii) The numbers $h_{a}^{-}(M): \operatorname{ext}_{A}^{-}(A / a, M), \operatorname{tor}_{-}^{A}(A / a, M)$ are finite simultaneously.

In that case, $\operatorname{ext}_{A}^{-}(A / a, M)+\operatorname{tor}_{-}^{A}(A / a, M) \leq n$.

(iii) If the numbers $h_{a}^{-}(M), \operatorname{ext}_{\Lambda}^{-}(A / a, M), \operatorname{tor}_{-}^{A}(A / a, M)$ are inflnite; then, for any ideal $a^{\prime}$, open in the a-adic topology of $A$, the numbers $\operatorname{ext}_{A}^{-}\left(A / a^{\prime}, M\right), \operatorname{tor}_{-}^{A}\left(A / a^{\prime}, M\right), u_{-}^{a^{\prime}}(M)$ are also infinite.

(iv) If $f: A \rightarrow B$ is a morphism of rings, if $b=f(a) B$, then, for each $B$-module $N$, we have $h_{b}^{-}(N)=\operatorname{ext}_{B}^{-}(B / b, N)=\operatorname{ext}_{A}^{-}(A / a, N)=$ $h_{a}^{-}(N), \operatorname{tor}_{-}^{B}(B / b, N)=\operatorname{tor}_{-}^{A}(A / a, N)$.

Proof:

(i) We have an isomorphism $K(x, M)^{\vee} \simeq K\left(x, M^{\vee}\right)$, so tor ${ }_{-}^{\wedge}\left(A / a, M^{\vee}\right)$ $=h_{-}\left(x, M^{\vee}\right)=h^{-}(x, M)=\operatorname{ext}_{A}^{-}(A \mid a, M)$.

(ii) This is a consequence of the self-duality of the Koszul complex: $H^{i}(x, M) \simeq H_{n-i}(x, M)$ (see $\left.[18,6.1 .8]\right)$.

(iii) When an ideal $a^{\prime}$ is open in the $a$-adic lopology, we have $a^{\prime} \supset$ $a^{r}$ for a certain natural number $r$. Using 1.6, we obtain $\infty=$ $\operatorname{tor}_{-}^{A}(A / a, M)=\operatorname{tor}_{-}^{A}\left(A / a^{r}, M\right) \leq \operatorname{tor}_{-}^{A}\left(A / a^{\prime}, M\right)=\infty$. The open ideal $a^{\prime}$ being fixed now, we have also $\operatorname{tor}_{-}^{A}\left(A / a^{\prime \prime}, M\right)=\infty$ for all ideals $a^{\prime \prime}$, open in the $a^{\prime}$-adic topology. So the module $M$ belongs to the class $\mathcal{C}_{a^{\prime}}$ (1.4) and, as $M=a^{\prime} M$, we have also $U_{0}^{a^{\prime}}(M)=0(1.3)$ and $u_{-}^{a^{\prime}}(M)=\infty$.

(iv) Take the image $y$ in $B$ of the sequence $x$ generating $a: y_{i}=f\left(x_{i}\right)$. There are obvious isomorphisms $K .(y) \simeq K .(x) \otimes_{A} B, K(y) \otimes_{B}$ $N \simeq K(x) \otimes A N, \operatorname{Hom}_{B}(K,(y), N) \simeq \operatorname{Hom}_{A}(K .(x), N)$. So this is another consequence of the theorem above.

Note that we have obtained here a change of rings result for the depth $h_{a}^{-}(\cdot)$ (a finitely generated) in a situation where we don't have a change of rings theorem for the local cohomology functors $H_{a}^{i}(\cdot)$.

\section{2. $U$-codepth}

In local cohornology, we have the equality $h_{a}^{-}(M)=\operatorname{ext}_{A}(A / a, M)$ already mentionned (1.6). We want an analogous result for the $U$-codepth 
$u_{-}^{a}$ using Tor instead of Ext. To achicve this, we need some preparation.

2.1.

The following lifting proposition was proved in $([\mathbf{4}, 3.5])$ in the local case.

Proposition. Let a be an ideal contained in the Jacolson radical of the ring $A$, and let $F$ be a flat $A$-module such that $F / a F$ is free as an $A / a$-module. If $\left\{e_{i} \mid i \in I\right\}$ is a set of elements of $F$ such that its image $\left\{\bar{e}_{i} \mid i \in I\right\}$ in $F / a F$ is a basis of $F / a F$, then the set $\left\{e_{i} \mid i \in I\right\}$ generates a pure free submodule $L$ of $F$, and $F=L+a F$.

\section{Proof:}

We first prove the freeness of the $e_{i}$ in $F$. If $\sum_{i=1}^{m} b_{i} e_{i}=0, b_{i} \in A$, we put $b=\left(b_{1}, \ldots, b_{m}\right), e=\left(e_{1}, \ldots, e_{m}\right)$; in matricial langutge, wo have $b . e^{t}=0$. By a flatness criterium $([5$,$] , Section 2$, proposition 13 , corollary 1]), there is a matrix $X \in A^{m \times n}$ and a vector $\int \in F^{1 \times n}$ such that $e^{l}=X \cdot f^{t}, b . X=0$. Deroting the images modulo the ideal a by $\left(^{-}\right)$, we have $\bar{e}^{t}=\bar{X} \cdot \bar{f}^{t}$. But the $\ddot{e}_{i}$ form a basis of $F / a F$, the matrix $\bar{X}$ is thus right-invertible, and so is the matrix $X$ since $a$ is contained in the Jacobson radical of $A$. From $b . X=0$ we deduce $b=0$ and the freeness of the $e_{i}$ in $F$.

We now prove the purity of $L$ in $F$. As $F$ is flat, it is enough to check the injectivity of the maps $L / c L \rightarrow F / c L$ for each ideal $c$ of $A$. As the image $\bar{e}_{i}$ of the elements $e_{i}$ of $L$ form a basis of $F / a F$, the natural morphism $L / a L \rightarrow F / a F$ is an isomorphism, and so is $L /(a+c) L \rightarrow$ $F /(a+c) F$. But the ideal $(a+c) / c$ of $A / c$ is contained in the Jacobson radical of $A / C$. We apply the first part of the proof to the flat $A / C$-module $F / c F$ and to the images of the $e_{i}$ in $F / c F$ : these images generate a free submodule of $F / c F$, so the morphism $L / c L \rightarrow F / c L$ is injective and $L$ is pure in $F$. Now $F=L+a F$ is clear.

\section{2 .}

Proposition. Let a be an ideal contained in the Jacobson radical of the ring $A$, and let $M$ be an $A$-module with $M=a M$. Then there exists an epimorphism $P \rightarrow M$ where $P$ is a flat $A$-module with $P=a P$.

\section{Proof:}

Let $0 \rightarrow K \rightarrow F \rightarrow M \rightarrow 0$ be an exact sequence, where $F$ is free. As $M=a M$, the sequence $K / a K \rightarrow F / a F \rightarrow 0$ is exact. Choose in $K$ elemerts $y_{i}$ whose images in the free $A / a$-module $F / a F$ form a besis 
of $F / a F$. By 2.1, these $y_{i}$ generate a free pure submodule $L$ of $F$, and $L \subset K$. So $P=F / L$ is flat, and $P=a P$ since $F=L+a F$. Now the epimorphism $F \rightarrow M$ induces an epimorphism $P=F / L \rightarrow M$.

\section{3.}

In the preceding proposition, the condition $M=a M$ means that the Tor-codepth and the $U^{a}$-codepth of $M$ are positive: $\operatorname{tor}_{-}^{A}(A / a, M)>$ $0, u_{-}^{a}(M)>0(1.3)$. On the other hand, for the flat module $P$, we have $\operatorname{tor}_{-}^{A}(A / a, P)=\infty=u_{-}^{a}(P)\left(P\right.$ belongs to $\mathcal{C}_{a}$, see 1.4$)$. So this shows that the functions $\operatorname{tor}_{-}^{A}\left(A / a_{1}.\right)$ and $u_{-}^{a}(\cdot)$ satisfy the duals of the axions of Itoh characterizing a homological grade [9]. It will be used to prove the equality between the $U^{a}$-codepth and the Tor-codepth.

When the ring is noetheriarl, we get rid of the assumption on the ideal $a$ by tensorizing with $\hat{A}$. Indeed, in that case, $\hat{A}$ is $A$-flat, $\hat{a}_{n}=a^{n} \hat{A}, \hat{a}$ is contained in the Jacobson radical of $\hat{A}[\mathbf{1}]$, and we have the following easy observation, extending $([\mathbf{1 8}, 2.2 .2])$.

Lemma. Let a be an ideal of the noetherian ring $A$. Then, for each $A$-module $M$, the module $\hat{M}$ is isomorphic to the $\hat{a}$-adic completion of the $\hat{A}$-module $\hat{A} \otimes_{A} M$, and $U_{i}^{a}(M) \simeq U_{i}^{\hat{a}}\left(\hat{A} \otimes_{A} M\right), \operatorname{Tor}_{i}^{A}\left(A / a^{n}, M\right) \simeq$ $\operatorname{Tor}_{i}^{\hat{A}}\left(\hat{A} / \hat{\Omega}^{n}, \hat{A} \otimes_{A} M\right)$ for all $n>0$.

(If $L . M \rightarrow 0$ is a free resolution of $M$, then $\hat{A} \otimes_{A} L$ is a free resolution of the $\hat{A}$-module $\hat{A} \otimes_{A} M$, and $\hat{A} / \hat{a}^{n} \otimes_{\hat{A}}\left(\hat{A} \otimes_{A} L.\right) \simeq \hat{A} / \hat{a}^{n} \otimes_{A}$ $L . \simeq A / a^{n} \otimes_{A} L$. This gives the result, after taking limits for the $U$-part of it).

\section{4 .}

Theorem. Let a be an ideal of the ring $A$. If $a$ is contained in the Jacobson radical of $A$ or if $A$ is noetherian, then, for each $A$-module $M, u_{-}^{a}(M)=\operatorname{tor}_{-}^{A}(A / a, M)$.

\section{Proof:}

We already know that $u_{-}^{a}(M)$ and $\operatorname{tor}_{-}^{A}(A / a, M)$ vanish simultaneously, exactly when $M \neq a . M(1.3)$.

With 2.3 , we are reduced to the first case, where $a$ is contained in the Jacobson radical of $A$. In that case, if one of the numbers above is positive finite, we have an exact sequence $0 \rightarrow M_{1} \rightarrow P \rightarrow M \rightarrow 0$, where $P$ is flat and $P=a P(2.2)$. The long exact sequences associated with it shows $\operatorname{tor}_{-}^{A}\left(A / a_{1}, M_{1}\right)=\operatorname{tor}_{-}^{A}(A / a, M)-1, \cdot u_{-}^{a}\left(M_{1}\right)=u_{-}^{a}(M)-1$. So 
by an induction argument we have $u_{-}^{a}(M)=\operatorname{tor}_{-}^{A}(A / a, M)$. This shows also that these two numbers are infinite simultaneously.

\section{5 .}

Proposition. Under the hypothesis of 2.4, if $t=u_{-}^{u}(M)<\infty$ and if the ideal a is finitely generaled, then

$$
U_{t}^{u(}(M)^{\wedge}=\lim ^{t} \operatorname{Tor}_{t}^{\Lambda}\left(A / a^{7 t}, M\right)
$$

\section{Proof:}

This is done by induction on $l$, using an exact sequence as in 2.4 (after having tensored by $\hat{A}$ in the noctherian case), the case $t=0$ is 1.3 .

\section{3. $U$-dimension and $H$-dimension over a noetherian ring}

We now study the dimensions $u_{+}^{u}(M)$ and $h_{a}^{+}(M)$ as defined in 1.6 . Our rings are now noetherian.

In local cohomology, it is known that $h_{t}^{+}(M) \leq \operatorname{dim} M$ for each $A$ module $M$ [15] (moreover, if $M$ is finitely generated and if $a=m$ is the maximal ideal of a local ring; then $h_{m}^{+}(M)=\operatorname{dim} M$ [11]).

We stablish an analogous inequality for the $U$-dimension. This in turn allows us to refine the inequality above. To achieve this, we need a change of rings theorcm for the $U_{i}^{n}$, analogous to the corresponding one in local cohomology.

\section{1 .}

Let $M_{i}, i \in I$, be a family of modules over the noetherian ring $A$.

Then $\left(\oplus_{i} M_{i}\right)^{\wedge}=\left\{w \in \mathrm{ll}_{i} \hat{M}_{i} \mid\right.$ for all $n$, wll but finitely many components $w_{i}$ of $w$ belong to $\left.a^{n} \hat{M}_{i}\right\}([16,9.4])$, so $\left(\oplus_{i} M_{i}\right)^{\wedge}=\left(\oplus_{i} \hat{M}_{i}\right)^{\wedge}$.

When $M_{i} \simeq M$ for all $i$, we write as usual $M^{(I)}=\oplus_{i} M_{i}, M^{I}=\Pi_{i} M_{2}$.

The following lemma was observed in $([14$, p. 77. 2.4.2]).

Lemma. Let $I$ be a set. Each short exact sequence $0 \rightarrow M^{\prime} \rightarrow M \rightarrow$ $M^{\prime \prime} \rightarrow 0$ of finitely genernted modules over the noetherian ring $A$ gives rise to on exact sequence

$$
0 \longrightarrow\left(M^{\prime(l)}\right)^{\wedge} \stackrel{\hat{u}}{\longrightarrow}\left(M^{(I)}\right)^{\wedge} \stackrel{\bar{v}}{\longrightarrow}\left(M^{\prime \prime(l)}\right)^{\wedge} \longrightarrow 0
$$




\section{Proof:}

As $\hat{A}$ is $A$-flat and as $\hat{X}=\hat{A} \otimes_{A} X$ when $X$ is a finitely generated inodule, by tensorizing with $\hat{A}$ we are reduced to the case where $A$ and our finitely generated modules are complete in the a-adic topology. In that case, the sequence above is a restriction of the exact sequence $\theta \rightarrow$ $M^{\prime I} \rightarrow M^{I} \rightarrow M^{\prime \prime I} \rightarrow 0$, so $\hat{u}$ is injective. We already know that $\hat{v}$ is surjective (1.2). Let $w \in \operatorname{ker} \hat{v}=\left(M^{(I)}\right)^{\wedge} \cap M^{\prime}$. By the Artirt-Rees lemma, there is a natural number $c$ such that, for all $n \geq 0, a^{n+c} M \cap$ $M^{\prime}=a^{n}\left(a^{r} M \cap M^{\prime}\right)$. As $w \in\left(M^{(i)}\right)^{\wedge}$ : for each natural number $n$ there is a finite subset $J_{n}$ of $I$ such that, $\forall i \notin J_{n}, w_{i} \in a^{n+c} M$. So, $\forall i \notin J_{n}, w_{i} \in a^{n-1} c M \cap M^{\prime} \subset a^{n} M^{\prime}$, this means $w \in\left(M^{\prime(I)}\right)^{\wedge}$ and finishes the proof.

\section{Remark.}

By excrcising a little more, one can prove that $\left(M^{(I)}\right)^{\wedge}$ is in fact a pure $\hat{A}$-submodule of $\hat{M}^{\prime}$ when $M$ is finitely generated (see $[18,2.1 .9]$, for the case $M=A$ ). As a consequence, for all ideal $b$ of $A$, we have an isomorphism $\left(M^{(I)}\right)^{\wedge} / b \cdot\left(M^{(I)}\right)^{\wedge} \simeq\left((M / b M)^{(I)}\right)^{\wedge}$. Indeed, as $\hat{b}=b \hat{A}$ $([5$, Section 3,4 , corollary 1$])$, we are again reduced to the case where $A$ is complete. In that case, we apply the lerma to the sequence $0 \rightarrow$ $b M \rightarrow M \rightarrow M / b M \rightarrow 0$, we obtain $\left((b M)^{l}\right)^{\wedge}=\left(M^{(I)}\right)^{\wedge} \cap(b M)^{I}=$ $\left(M^{(I)}\right)^{\wedge} \cap b \cdot M^{I}=b \cdot\left(M^{(l)}\right)^{\wedge}$ and the desired isomorphism. In particular, for a free $A$-module $L$, we have $(L / b L)^{\wedge} \simeq \hat{L} / b \hat{L}$.

\section{2 .}

Proposition. Let $M$ be a finitely generated module over the noelleerian ring $A$, and let $I$ be a set. Then the module $N=M^{(I)}$ belongs to the classe $\mathcal{C}_{a}: U_{0}^{a}(N)=\hat{N}, U_{i}^{a}(N)=0$ for $i>0$.

\section{Proof:}

Let $\ldots L_{1} \stackrel{d_{1}}{\rightarrow} L_{0} \stackrel{d_{o}}{\rightarrow} M \rightarrow 0$ be a resolution of $M$, where the $L_{i}$ are finitely generated free. Put $M_{i}=\operatorname{im} d_{i}$. The short exact sequences $0 \rightarrow M_{i+1} \rightarrow L_{i} \rightarrow M_{i} \rightarrow 0$ give rise to exact sequences

$$
0 \longrightarrow\left(M_{i+1}^{(I)}\right)^{\wedge} \longrightarrow\left(L_{i}^{(I)}\right)^{\wedge} \longrightarrow\left(M_{i}^{(I)}\right)^{\wedge} \longrightarrow 0
$$

by 3.I. But $L^{(I)}$ is a free resolution of $M^{(I)}$, so we have the result. 


\section{3.}

Theorem. Let $f: R \rightarrow A$ be a morphism of noetherian rings such that $A$ is finitely generated as an $R$-module. Let $r$ be an ideal of $R$ and $a=f(r) A$ be its extension in $A$. Then $U_{i}^{r}$ and $U_{i}^{a}$ form naturally isomorphic descending connected exact sequences of functors from $A$ modules to $R$-modules.

Proof:

An $A$-module $M$ can be viewed as a $R$-module; on $M$, the $a$-adic and the $r$-adic topology are the same. Take a free resolution $\ldots L_{1} \rightarrow L_{0} \rightarrow$ $M \rightarrow 0$ of $M$ as an $A$-module. By (3.2), the modules $L_{i}$ belong to the classes $\mathcal{C}_{a}$ and $\mathcal{C}_{r}$ introduced in (1.4). Completing; we get the natural isomorphisms $U_{i}^{a}(M) \simeq H_{i}(\hat{L}) \simeq U_{i}^{r}(M)$.

\section{4 .}

The preceding theorem is useful for the computation of the $U_{i}^{a}(M)$, it brings us back to the first case studied by Matlis, namely the case where the ideal $a$ is generated by a regular sequence. Indeed, if $a=$ $\left(x_{1}, \ldots, x_{n}\right)$, we use a change of rings $B=A\left[X_{1}, \ldots, X_{n}\right] \stackrel{f}{\rightarrow} A$, where $B$ is a polynomial ring in the indeterminates $X_{i}$, where $f$ is defined by $f\left(X_{i}\right)=x_{i}$. The regular sequence $X_{1}, \ldots, X_{n}$ on the ring $B$ generates an ideal $b$ and, for each $A$-rnodule $M$, for all $i, U_{i}^{a}(M) \simeq U_{i}^{b}(M)$.

This allows generalizations of some Matlis results. Here is a first one (see $[13]$ ).

Corollary. If the ideal a is generated by a sequence $x_{1}, \ldots, x_{n}$ regular on the module $M$, then $M$ belongs to $\mathcal{C}_{n}$.

\section{Proof:}

Lsing a change of rings as above, we are reduced to the case where the sequence $x=x_{1}, \ldots, x_{n}$ is regular on both $A$ and $M$. The sequence $x^{t}=x_{1}^{t}, \ldots, x_{n}^{t}$ generate an ideal $a_{t}$, the Koszul complex $K .\left(x^{t}\right)$ is a finite free resolution of $A / a_{l}$, and we have $H_{i}\left(x^{l}, M\right)=\operatorname{Tor}_{i}^{A}\left(A / a_{t}, M\right)=0$ for all $i>0$ since the sequence $x^{l}$ is regular on $M$. As noted in (1.4) this implies $M \in \mathcal{C}_{a}$.

\section{5 .}

We say that an ideal $a$ of the noetherian ring $A$ can be generated by $n$ elements up to radical equivalence if there exist clements $x_{1}, \ldots, x_{n}$ generating an ideal $b$ with $\operatorname{rad} a=\operatorname{rad} b$. Then the $a$-adic and the $b$-adic topology are the same, and $U_{i}^{a}(M)=U_{i}^{b}(M)$. 
Assume $a=\left(x_{1}, \ldots, x_{n}\right)$. Matlis proved that $U_{i}^{a}(M)=0$ for $i>n$ if the sequence $x_{1}, \ldots, x_{n}$ is regular on the ring $A$ ([12, theorem 4.12]). Greenless and May obtained the same result in a more general situation. The change of rings theorem allows a slight refinement of this. However, Matlis method dualized gives also a very elementary proof of the corresponding result in local cohomology, in a somewhat unusual formulation which will be useful later. That is why we insert it here.

Lemma. Let $M$ be a module over the noetherian ring $A$. If the image of the ideal a in $A / \mathrm{Ann}_{A} M$ can be generated by $n$ elements up to radical equivalence, then $u_{+}^{a}(M) \leq n$ and $h_{a}^{+}(M) \leq n$.

\section{Proof:}

We first use a change of rings $A \rightarrow A / \mathrm{Ann}_{A} M$, then a change of rings as clescribed in 3.4, and we are reduced to the case where the ideal $a$ is generated by a regular sequence $x_{1}, \ldots, x_{n}$. Write again $a_{t}=$ $\left(x_{1}^{t}, \ldots, x_{n}^{t}\right)$. As $A / a_{t}$ is of finite projective dimension $n$ and as $H_{a}^{i}(M)=$ $\lim _{\rightarrow} \operatorname{Ext}_{A}^{i}\left(A / a_{t}, M\right)([15]$, or $[18,4.1 .3])$, we have $H_{a}^{i}(M)=0$ for $i>n$.

Dually, following Matlis, we take a projective resolution of $M \ldots P_{i} \stackrel{d_{i}}{\rightarrow}$ $\cdots \rightarrow P_{0} \rightarrow M \rightarrow 0_{2}$ and put $M_{n}=\operatorname{im} d_{n}$. For $k \geq 1$, we have $\operatorname{Tor}_{k}^{A}\left(A / a_{t}, M_{n}\right) \simeq \operatorname{Tor}_{k+n}^{A}\left(A / a_{t}, M\right)=0$, so $M_{n} \in \mathcal{C}_{a}(1.4)$ and $u_{+}^{a}(M) \leq$ $n$.

\section{6.}

Here is another generalization of a Matlis result.

Corollary. In the situation of $(3.5), U_{n}^{a}(M) \simeq U_{n}^{n}\left(H_{a}^{0} M\right)$.

\section{Proof:}

Followirig Matlis ([12, corollary 5.5]), write $N=M / H_{a}^{0}(M)$ and take an injective hull $J$ of $N$ vicwed as an $A / \mathrm{Ann}_{A} M$-module. By (3.5), we have cxact sequences

$$
\begin{aligned}
& 0 \longrightarrow U_{n}^{a}\left(H_{a}^{0} M\right) \longrightarrow U_{n}^{a}(M) \longrightarrow U_{n}^{a}(N) \\
& 0 \longrightarrow U_{n}^{a}(N) \longrightarrow U_{n}^{a}(J)
\end{aligned}
$$

Let $\bar{a}$ be the image of $a$ in $A / \operatorname{Ann}_{A} M$. As $H_{a}^{0}(N)=0$, we have also $H_{\bar{a}}^{0}(J)=0$ and $h_{\bar{a}}^{-}(J)=\infty$ since $J$ is an injective $A / A_{A n n} M$-module. Using $\left(1.7\right.$ corollary), we obtain $h_{\Omega}^{-}(J)=\infty=u_{--}^{a}(J)$. So $U_{h}^{u}(J)=$ $0, U_{n}^{a}(N)=0$, and the result follows from the first exact sequence. 


\section{7.}

Cornbining both local cohomology and local homology, we sharpen the bounds obtained in 3.5 for the $U$-dimension and the $H$-dimension.

Theorem. Let a be an ideal of the noetherian ring $A$ and $M$ an $A$ module. If the image of the ideal a in $A / A^{\prime} n_{A} M$ can be generated by $n$ elements up to radical equivalence, if $h_{i}^{-}(M)$ is finite, then

(i) $u_{+}^{a}(M)+h_{a}^{-}(M) \leq n$,

(ii) $h_{t a}^{+}(M)+u_{-}^{a}(M) \leq n$ if a is contained in the Jacobson radical of the ring $A$.

Proof:

Renark first that these inequalities are not equivalerit, modules are not necessarily duals.

Let us first prove (i).

If $h_{a}(M)=0$, this is $(3.5)$.

If $h_{a t}^{-}(M)=t>0$, we go by induction on $t$, using an exact sequence: $0 \rightarrow M \rightarrow J \rightarrow M_{1} \rightarrow 0$, where $J$ is an injective hull of $M$ viewed as an $A / \mathrm{Ann}_{A} M$-module. As in 3.6, wc have $h_{f-}^{-}(J)=\infty=u_{-}^{a}(J)$, so $h_{a}^{-}\left(M_{1}\right)=h_{a}^{-}(M)-1, u_{+}^{a}\left(M_{1}\right)=u_{+}^{a}(M)+1$; we add these equalities and obtain (i) by induction.

We now prove (ii) in a dual way.

If $u_{-}^{a}(M)=0$, this is (3.5). In any case, $u_{-}^{a}(M)<\infty$ (use 2.4 and 1.7 corollary ii). If $u_{-}^{a}(M)=t>0$, we use an exact sequence $0 \rightarrow M_{1} \rightarrow$ $P \rightarrow M \rightarrow 0$, where $P$ is a flat $A / \operatorname{Ann}_{A} M$-module with $P=a_{a} P(2.2)$. We write $\bar{a}$ for the image of the ideal $a$ in the ring $\bar{A}=A / \operatorname{Ann}_{A} M$. We have $\infty=\operatorname{tor}_{-}^{\bar{A}}(\bar{A} / \bar{a}, P)=\operatorname{tor}_{-}^{A}\left(A / a_{1}, P\right)=u_{-}^{a}(P)(1.7$ corollary (iv) and 2.4), $h_{a}^{-}(P)=\infty$ (1.7 corollary ii). The long exact sequences of the $U_{i}^{\prime \prime}$ and $H_{a}^{i}$ give thus $u_{-}^{\prime a}\left(M_{1}\right)=u_{-}^{a}(M)-1, h_{a}^{+}\left(M_{1}\right)=h_{a}^{+}(M)+1$. The conclusion follows now by induction.

\section{Remark.}

Let $a$ be a fuilely generated ideal of an arbitrary commutative ring $A$, and $n$ a natural number. If the $U^{2}$-global dimension of $A$ is less than $n$, i.e. if $U_{i}^{n}(N)=0$ for all $i>n$, for all modules $N$, then the conclusion in $(3.6,3.7$ (i) $)$ is still valicl.

Dually, if the $H_{a}$-global dimension of $A$ is less than $n$, i.e. if $H_{a}^{i}(N)=0$ for all $i>n$, for all modules $N$, then the conclusion in (3.7 ii) is validl.

Indeed, in the proofs of these facts the noetheriari hypothesis on the ring $A$ has been used only to oblixtin that the $U^{a}$-global dimension and the $H_{a}$-global dimension of $A$ are less than $n$ (3.5). 
3.8.

The last results are more suggestive in the local case.

Theorem. Let $A$ be a noetherian local ring of macimal ideal $m$, and $N$ an $A$-module. Then

(i) $u_{*}^{7 n}(M) \leq \operatorname{dim} A / \operatorname{Ann}_{A} M-h_{u^{-}}^{-}(M)$

(ii) $h_{a}^{+}(M) \leq \operatorname{dim} A / \operatorname{Ann}_{A} M-u_{-}^{a}(M)$

Note that we cannot replace $\operatorname{dim} A / \operatorname{Ann}_{A} M$ by $\operatorname{dim} M$.

\section{Example.}

Let $M=E$ be the injective hull of the residue field of a complete local ring $A$ of dimension $d>0$. We know $\operatorname{dim} E=0, \operatorname{Ann}_{A} E=0 . E \simeq A^{\vee}$, so $U_{i}^{m}(E) \simeq H_{m}^{i}(A)^{\vee}(1.5)$. As $H_{m t}^{d}(A) \neq 0$, we have $u_{+}^{m}(E)=d$. We have also $u_{-}^{m}(E)=h_{m}^{-}(A)$, the classical depth of the ring $A$, and $h_{m}^{-}(E)=0=h_{m}^{+}(E)$.

Note also that these inequalities might be strict.

\section{Example.}

In $([\mathbf{1 6}, 9.4])$, we showed a complete module $M$ over a regular local ring of dimension $d>1$, such that $\operatorname{Ann}_{A} M=0, \operatorname{Supp} M=\operatorname{Spec} A$ (so that $\operatorname{dim} M-\operatorname{dim} A$ ), and such that $h_{m}^{-}(M) \leq h_{m}^{+}(M)<d$. As that module is complete, we have also $u_{-}^{m}(M)=0=u_{+}^{m}(M)$ (see 1.4, $M \in \mathcal{C}_{m}$ ). For that module both inequalities are strict.

\section{Questions.}

1. How can we refire the above inequalities?

2. If $M$ is artinian, then $u_{+}^{m}(M)=\operatorname{dim} A / \mathrm{Ann}_{A} M$. Is this still true for a module $M$ with $M=H_{m}^{0}(M)$ ?

\section{References}

1. M. F. AtiYah AND 1. G. Macdonald, "Introduction to commutative algebra," Addison-Wesley, 1969.

2. S. BARger, A theory of grade for commutative rings, Proc. of the A.M.S. 36(2) (1972), 365-368.

3. 3. BARTIJN, Flatness, completions, regular sequences, un ménage à trois, Thesis, Utrecht, 1985.

4. J. BARTIJN AND J. R. STROOKER, "Modifications monomiales;" Semin. Dubreil-Malliavin, Lect. Notes in Math. 1029, SpringerVerlag, 1983, pp. 192-217.

5. N. Bourbaki, "Algèbre commutative," Hermann, 1961. 
6. H. Cartinn and S. Embenberc, "Hotnological algebra," Princeton Math. Ser. 19, Princeton University Press, 1956.

7. J. P. C. Greenless and J. P. MaY; Derived functors of $I$-adic completion and local cohomology, to appear in Joumal of Algebro.

8. M. Hochster, Grade-sensitive modules and perfect modules, Proc. London Math. Soc., III, Ser. 29 (1973), 25-43.

9. S. FroH, Aximnatic charactcrizations of grade for commutative rings, Hiroshima Math. J. 8 (1978), 91-100.

10. D. KIRBY AND H. E. MBHFAn, The homological grade of a module, Mathematika 35 (1988), 114-125.

11. I. G. Macdonatd And R.Y. Shatr, An elementary proof of the non-vanishing of certain local cohomology modules, Q.J. Wath. Oxf. II, Ser. 23 (1972), 197-204.

12. E. Matuls, The Koszul complex and duality, Comm. in Alg. 1 (1974), 87-144.

13. E. MATuIs, The higher properties of R-sequences, J. Algebra 50 (1978), 77-122.

14. M. Rayanud ê L. Grison. Critères de platitude et de projectivité, Invent. Math. 13 (1971), 1-89.

15. R. Y. SHARP, Local cohomology theory in commutative algebra, Q.J. Math, Oxf. H, Ser. 21 (1979), 425-434.

16. A. M. Smon, Some homological properties of complete modules, Math. Proc. Camb. Phil. Soc. 108 (1990), 231-246.

17. I. R. STrooker, "A general acyclicity lemma and its uses," Topics in Algebra, Banach Center Publ. 26, part 2, Warsaw, 1990, pp. 229-233.

18. J. R. Strooker, "Homological questions in local algebra," London Math. Soc., Lect. Note Ser. 145, Cambridge Univ. Press, 1990.

Université Libre de Bruxciles

Campus Plainc, C.P. 211

Boulevard du Triomphe

B-1050 Bruxelles

BELGIQUE 